\title{
Interstitial lung disease and bronchiectasis in children with primary immunodeficiencies: a single-centre experience
}

\author{
Katarzyna Tąpolska-Jóźwiak, Aleksandra Szczawińska-Popłonyk, Anna Bręborowicz \\ Department of Pediatric Pneumonology, Allergology and Clinical Immunology, Poznan University of Medical Sciences, Poland
}

\section{ABSTRACT}

\begin{abstract}
Introduction: Primary immunodeficiencies (PIDs) are a heterogeneous group of inherited disorders in which one or several components of the immune system are involved. Pulmonary complications remain the most common cause of morbidity and mortality in patients with PIDs. The term chronic lung disease refers to interstitial lung disease (ILD) and bronchiectasis. Infectious as well as non-infectious factors may contribute to the development of chronic lung disease. In contrast to adults with PIDs, reports about chronic lung disease in children are limited. Prevention and early diagnosis of pulmonary complications improve patients' prognosis and quality of life.

Aim of the study: To evaluate ILD and bronchiectasis in immunocompromised children.

Material and methods: The study was conducted through a retrospective review of the data of 44 children with different PIDs, taking into consideration the following: pulmonary infections, autoimmunity, presence of comorbidities, and therapy performed before the diagnosis of ILD/bronchiectasis.

Results: Interstitial lung disease was diagnosed in 25 cases (56.82\%) and bronchiectasis in 8 cases (18.18\%); both diseases were observed in 11 cases (25.00\%). The earliest radiological features of ILD were detected in patients with combined PIDs. Bronchiectasis had already been reported in children above 5 years of age with a defect in antibody production. Bacterial infections requiring intravenous antibiotic treatment occurred significantly more frequently in the medical history of children with bronchiectasis $(p<0.001)$, whereas children with the ILD component showed a higher prevalence of pulmonary fungal infections $(p=0.034)$.

Conclusions: Regular clinical evaluation, supported by additional tests results including radiological findings, is crucial for the early diagnosis of chronic lung disease.
\end{abstract}

\section{KEY WORDS:}

children, primary immunodeficiency, interstitial lung disease, bronchiectasis, pulmonary complications.

\section{INTRODUCTION}

Primary immunodeficiencies (PIDs) are a heterogeneous group of inherited disorders in which one or several components of the immune system are involved. The spectrum of respiratory manifestations comprises conditions which concern either the upper airways or the lower respiratory tract. The latter are considered to be clinically more important and more specific for PIDs. Pulmonary complications remain the most common cause of morbidity and mortality in these patients, and what is more, they are usually the first presentation of the disease [1-3]. The term chronic lung disease refers to both interstitial lung disease (ILD) and bronchiectasis $[4,5]$.

Interstitial lung disease encompasses a group of various disorders involving pulmonary parenchyma and which interfere with gas exchange. The aetiology of ILD in PIDs remains unclear to some extent. Although there have been reports about post-infectious ILD, it has recently been considered the result of immune

\section{ADDRESS FOR CORRESPONDENCE:}

Katarzyna Tąpolska-Jóźwiak, Department of Pediatric Pneumonology, Allergology and Clinical Immunology, Poznan University of Medical Sciences, Szpitalna 27/33, 60-572 Poznan, Poland, e-mail: ktapolska@wp.pl 
dysregulation $[2,3]$. Chronic non-infectious complications are best characterized in common variable immunodeficiency (CVID), in which a specific entity called granulomatous-lymphocytic interstitial lung disease (GLILD) is observable [5].

Bronchiectasis is defined by a progressive injury of airway walls and is believed to be the result of chronic or repeated airway inflammation, resulting in irreversible bronchodilation [6]. According to the latest reports, bronchiectasis secondary to PIDs in childhood is not always a progressive condition, if appropriate treatment is performed [7].

Computed tomography (CT), especially high-resolution computed tomography (HRCT), in conjunction with pulmonary functions tests, plays an essential role in the identification and monitoring of pulmonary complications; however, other imaging methods like chest X-ray (CXR), magnetic resonance imaging (MRI), or lung ultrasound may be useful [2].

In contrast to adults with PIDs, reports about chronic lung disease in children are limited. The main aim of this study is to investigate ILD and bronchiectasis in children with different PIDs, to contribute to a better understanding of the clinical course of PIDs and, in consequence, to improve the early detection and prevention of chronic lung disease.

\section{MATERIAL AND METHODS}

\section{STUDY GROUP}

This study was conducted through a retrospective review of the medical records of 450 patients referred to the department of paediatric pneumonology, allergology, and clinical immunology in 2011-2019 due to the suspicion of PID. Children with a confirmed diagnosis of PID and chronic lung disease were selected. We excluded patients with other conditions, such as cystic fibrosis, primary ciliary dyskinesia, and bronchopulmonary dysplasia. Patients with haemodynamically significant congenital heart disease were excluded, except for children with DiGeorge syndrome (DGS), of which congenital heart disease is an integral feature.

Overall, 44 children ( $9.78 \%$ of the entire group) were included in the analysis.

\section{METHODS}

In each case we reviewed radiology reports searching for the presence of changes characteristic of ILD and bronchiectasis. In 40 cases CT was performed; in the others an MRI technique was used because of increased radiation sensitivity.

We collected data from the medical histories and assigned them to the following categories: General characteristics, comprising the following: sex, primary immuno- deficiency (diagnoses were made according to Working Definitions for Clinical Diagnosis of PID established by the European Society of Immunodeficiencies [8]. Then we grouped PIDs in alignment with the 2019 Update on the Classification from the International Union of Immunological Societies Expert Committee [9]) and age at the recognition of ILD/bronchiectasis

Radiological findings, dividing patients into 3 groups: patients with radiological features of 1) ILD; 2) bronchiectasis (BE); or 3) both diseases (ILD + BE).

Medical history of the patient, including the following: 1) respiratory tract infections (recurrence, verified viral infections requiring hospitalization, bacterial infections requiring intravenous antibiotic therapy, fungal lung infections); 2) autoimmunity; 3) other comorbidities; 4) therapy performed before diagnosis of ILD/bronchiectasis, including treatment with immunoglobulin replacement therapy (IRT), antibiotics, inhaled corticosteroids, immunosuppressive therapy.

\section{CALCULATIONS AND STATISTICAL ANALYSIS}

To evaluate the presence of serious pulmonary complications, we used a percentage distribution of the collected medical data.

Statistical analysis was conducted to assess the association between selected clinical aspects and radiological findings. We focused on the influence of the following: 1) recurring infections; 2 ) verified viral infections requiring hospitalization; 3 ) bacterial infections requiring intravenous antibiotic therapy; 4) fungal lung infections, and 5) autoimmunity on the development of lung disease in each group - ILD, BE, and ILD + BE. Given the unequal group number, we used the method of weighting. The statistical analysis was conducted on the basis of the $\chi^{2}$ test and Fischer's exact test when the $\chi^{2}$ test's assumptions were not met. A $p$-value $<0.05$ was considered as significant.

\section{RESULTS}

\section{GENERAL CHARACTERISTICS}

We evaluated 44 immunodeficient children - 28 male and 16 female, aged between 2 weeks and 17 years (median age 6.5 years). Chronic lung disease was most common in children with profound immunodeficiency affecting cellular and humoral immunity - ILD occurred in 25 children (including 11 with combined immunodeficiency - CID) and bronchiectasis in 12 patients (including 5 with CID). The earliest radiological features of ILD were detected in the first year of life. In PIDs with the predominant antibody defect, bronchiectasis was reported above 5 years of age; this diagnosis was made in 7 patients, whereas radiological features of interstitial lung disease were found in 9 children. Figure 1 presents the distribution of age in connection with the recognition 
ILD bronchiectasis —ILD + bronchiectasis

$\square$ one grid means one patient

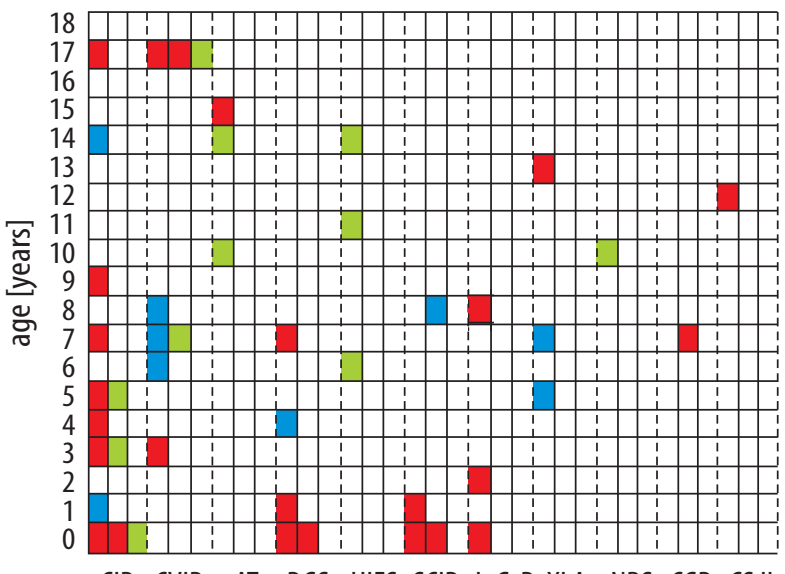

CID CVID AT DGS HIES SCID IgGSD XLA NBS CGD CS II

FIGURE 1. Distribution of age [in years] at the time of recognition of ILD/bronchiectasis in relation to primary immunodeficiency
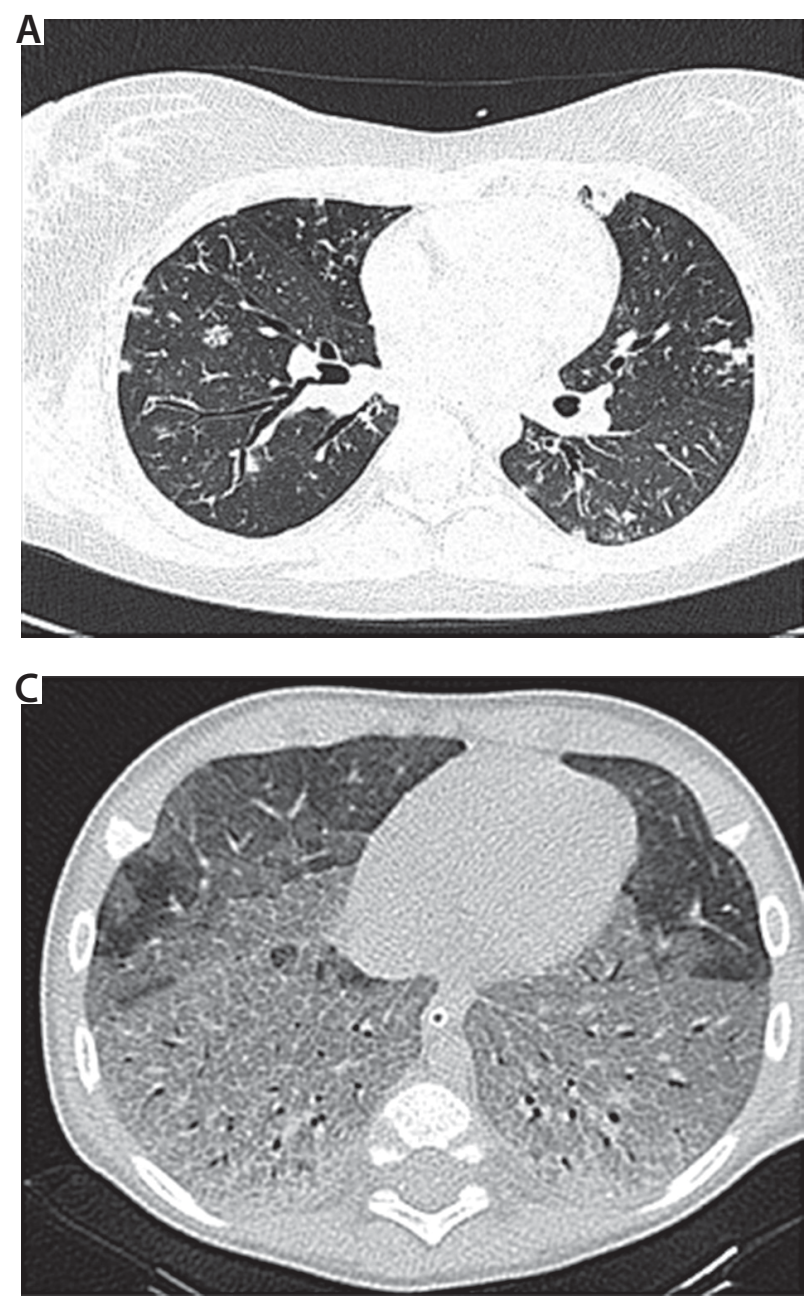

FIGURE 3. Chest transaxial CT images in a lung window of: A) a 14-year-old girl with HIES: bilateral diffuse nodular opacities with branching micronodular opacities (tree-in-bud sign), diffuse airway wall thickening, cylindrical bronchiectasis; B) a 12-year-old girl with GS II: multiple bilateral parenchymal and subpleural nodular opacities (lymphomatoid granulomatosis); C) a 5-month-old boy with SCID: large patchy areas of ground-glass opacity and mosaic perfusion, interlobular septal thickening; D) chest transaxial contrast-enhanced T1-weighted MRI image of a 9-year-old boy with CID: bilateral diffuse nodular opacities (granulomas, suspected GLILD) and hilar lymphadenopathy 
TABLE 1. Factors from the medical history of children with ILD and bronchiectasis

\begin{tabular}{|l|c|c|c|c|c|}
\hline & $\begin{array}{c}\text { In total } \\
(n=44)\end{array}$ & $\begin{array}{c}\text { ILD } \\
(n=25)\end{array}$ & $\begin{array}{c}\text { BE } \\
(n=8)\end{array}$ & $\begin{array}{c}\text { ILD }+ \text { BE } \\
(n=11)\end{array}$ & $p$ \\
\hline Recurrent respiratory tract infections & $\begin{array}{c}86.36 \% \\
(n=38)\end{array}$ & $\begin{array}{c}84.00 \% \\
(n=21)\end{array}$ & $\begin{array}{c}100 \% \\
(n=8)\end{array}$ & $\begin{array}{c}81.82 \% \\
(n=9)\end{array}$ & $0.067^{\mathrm{b}}$ \\
\hline Viral infection requiring hospitalization* & $\begin{array}{c}36.36 \% \\
(n=16)\end{array}$ & $\begin{array}{c}40.00 \% \\
(n=10)\end{array}$ & $\begin{array}{c}25.00 \% \\
(n=2)\end{array}$ & $\begin{array}{c}36.36 \% \\
(n=4)\end{array}$ & $0.458^{\mathrm{a}}$ \\
\hline Bacterial infections with intravenous antibiotic therapy** & $\begin{array}{c}59.09 \% \\
(n=26)\end{array}$ & $\begin{array}{l}40.00 \% \\
(n=10)\end{array}$ & $\begin{array}{c}87.50 \% \\
(n=7)\end{array}$ & $\begin{array}{c}81.82 \% \\
(n=9)\end{array}$ & $<0.001^{\text {a }}$ \\
\hline Fungal lung infections*** & $\begin{array}{c}18.18 \% \\
(n=8)\end{array}$ & $\begin{array}{c}24.00 \% \\
(n=6)\end{array}$ & $\begin{array}{c}0 \% \\
(n=0)\end{array}$ & $\begin{array}{c}18.18 \% \\
(n=2)\end{array}$ & $0.034^{\mathrm{b}}$ \\
\hline Autoimmunity**** & $\begin{array}{c}36.36 \% \\
(n=16)\end{array}$ & $\begin{array}{l}36.00 \% \\
(n=9)\end{array}$ & $\begin{array}{c}50 \% \\
(n=4)\end{array}$ & $\begin{array}{c}27.27 \% \\
(n=3)\end{array}$ & $0.261^{\text {a }}$ \\
\hline
\end{tabular}

*Epstein-Barr virus $(n=8)$, cytomegalovirus $(n=5)$, rhinovirus, influenza virus $(n=2)$, respiratory syncytial virus, herpes simplex virus type 1, human herpesvirus type 6, human bocavirus ( $n=1$ )

** Streptococcus pneumoniae $(n=9)$, Staphylococcus aureus $(n=8)$, Pseudomonas aeruginosa $(n=5)$, Escherichia coli $(n=5)$, Klebsiella sp. $(n=4)$, Stenotrophomonas maltophilia ( $(n=3)$,

Mycoplasma pneumoniae $(n=3)$, Haemophilus influenzae $(n=1)$, Proteus mirabilis $(n=1)$

*** Pneumocystis jirovecii $(n=6)$, Candida sp. $(n=2)$, Aspergillus sp. $(n=1)$

**** Thyroiditis $(n=4)$, cytopaenia $(n=3)$, hepatitis $(n=3)$, arthritis $(n=2)$, celiac disease $(n=2)$, polyneuropathy, dermatomyositis, skin granuloma, parotitis $(n=1)$

${ }^{a} X^{2}$ test, ${ }^{b}$ Fischer's exact test

$\angle D$ - interstitial lung disease, BE - bronchiectasis

TABLE 2. Comorbidities other than autoimmune diseases in children with ILD and bronchiectasis

\begin{tabular}{|l|c|c|c|c|c|c|c|c|}
\hline \multirow{2}{*}{ Comorbidity } & \multicolumn{2}{|c|}{ ILD } & \multicolumn{2}{c|}{ BE } & \multicolumn{3}{c|}{ ILD + BE } & \multicolumn{2}{c|}{ In total } \\
\cline { 2 - 11 } & $n$ & $\%$ ILD & $n$ & $\%$ BE & $n$ & $\%$ ILD + BE & $n$ & $\%$ \\
\hline Sinusitis & 6 & 24.00 & 2 & 25.00 & 3 & 27.27 & 11 & 25.00 \\
\hline Asthma & 6 & 24.00 & 0 & 0.00 & 4 & 36.36 & 10 & 22.73 \\
\hline Splenomegaly & 5 & 20.00 & 0 & 0.00 & 2 & 18.18 & 7 & 15.91 \\
\hline Chronic bronchitis & 1 & 4.00 & 3 & 37.50 & 2 & 18.18 & 6 & 13.64 \\
\hline Congenital heart disease & 3 & 12.00 & 1 & 12.50 & 1 & 9.09 & 5 & 11.36 \\
\hline Scoliosis & 2 & 8.00 & 0 & 0.00 & 2 & 18.18 & 4 & 9.09 \\
\hline Lymphadenopathy & 1 & 4.00 & 0 & 0.00 & 2 & 18.18 & 3 & 6.82 \\
\hline Gastroesophageal reflux disease & 3 & 12.00 & 0 & 0.00 & 0 & 0.00 & 3 & 6.82 \\
\hline Chronic stomatitis & 2 & 8.00 & 0 & 0.00 & 1 & 9.09 & 3 & 6.82 \\
\hline Hypopituitarism & 1 & 4.00 & 1 & 12.50 & 0 & 0.00 & 2 & 4.55 \\
\hline Osteoporosis & 1 & 4.00 & 0 & 0.00 & 1 & 9.09 & 2 & 4.55 \\
\hline
\end{tabular}

ILD - interstitial lung disease; $B E$ - bronchiectasis

of ILD/bronchiectasis and a PID diagnosis. The prevalence of PIDs is summarized in Figure 2.

\section{RADIOLOGICAL FINDINGS}

Interstitial lung disease was diagnosed in 25 (56.82\%), bronchiectasis in $8(18.18 \%)$, and features of both diseases in 11 cases $(25.00 \%)$. The vast majority of bronchiectasis was bilobar (17/19 cases), in all cases cylindrical and diffuse (Fig. 3).

\section{MEDICAL HISTORY OF THE PATIENTS}

Respiratory tract infections dominated in all groups, affecting all children with isolated bronchiectasis and over $80.00 \%$ with ILD component, but the difference was not statistically significant (Table 1). Viral infections requiring hospitalization as well as fungal lung infections were more characteristic for patients with ILD, whereas bacterial infections treated with intravenous antibiotic therapy were significantly more frequently reported in groups with bronchiectasis. There were no significant differences in the occurrence of autoimmune disorders between the groups.

Table 2 reviews other medical conditions recognized in the children in our study. It is apparent from this table that a considerable proportion of the children presented symptoms of sinusitis and asthma. Splenomegaly and asthma were present solely in patients with the ILD component, whereas chronic bronchitis occurred more often in children with bronchiectasis.

\section{TREATMENT}

Fourteen $(31.82 \%)$ of the children received IgRT, $25(56.82 \%)$ received long-term antibiotic treatment, 
and $21(47.73 \%)$ received inhaled corticosteroids. None of the patients received immunosuppressive therapy (including systemic corticosteroids). Complex treatment, containing immunoglobulin, antibiotics, and inhaled corticosteroids, was performed in 10 children (22.73\%). A quarter of the patients were left without pharmacological therapy before the diagnosis of ILD/bronchiectasis.

\section{DISCUSSION}

We proved that ILD and bronchiectasis may occur in immunodeficient children at any age. The earliest radiological features of ILD, as early as in the first year of life, were detected in patients with combined PIDs. In the group of children with a defect in the production of antibodies, bronchiectasis had already been reported in children above 5 years of age.

The study results indicate that profound PIDs affecting cellular and humoral immunity predispose most to ILD and bronchiectasis. Children with CID predominated, while children with SCID constituted a few per cent of the analysed group. SCID was related to ILD in infancy, which is consistent with earlier reports $[1,2]$ (Fig. 3A). Van der Burg et al. [10] described that the atypical form of SCID may also lead to bronchiectasis, recognized beyond 12 months of age, but none of the present subjects was diagnosed with it. Combined PIDs with associated or syndromic features, such as ataxia-telangiectasia (AT), DGS, hiper-IgE syndrome (HIES), and Nijmegen breakage syndrome (NBS), accounted for over a quarter of the analysed cases. Chronic lung disease in AT comprises the features of both phenotypes with overlapping consequences of neurological abnormalities, which is in line with our data. Similarly to cystic fibrosis, patients suffering from AT are exposed to chronic colonization of the respiratory tract by bacteria, which increases the risk of bronchiectasis, whereas ILD may be seen in up to a quarter of patients $[11,12]$. Although pulmonary disease is not a major presentation in children with DGS, lung complications are common $[1,13]$. Recurrent pyogenic pneumonia, commonly resulting in bronchiectasis and pneumatoceles formation, is a typical pulmonary manifestation of HIES. Damage to the lung tissue leads to interstitial lesions, resulting in a characteristic picture of chronic lung disease [14]. In our study, patients presented a high degree of destruction of lung tissue at the time of diagnosis of HIES (Fig. 3C).

The second most numerous group were patients with predominant antibody deficiencies. CVID was associated with ILD, bronchiectasis, and mixed variant. Although CVID is the most prevalent symptomatic PID, and chronic lung disease is among its most common complications, very little attention has been paid to the paediatric population so far [15]. Ramzi et al. [16] in their meta-analysis reported that the overall prevalence of bronchiectasis in CVID patients was 34\%, while Van de Hen et al. [17] highlighted the occurrence of bronchiectasis in 20\% of children with CVID, ILD in $15 \%$, and $5 \%$ patients showed a mixed pattern. Granulomatous-lymphocytic interstitial lung disease encompasses granulomatous and lymphoid infiltration, which in an extrathoracic location may mimic sarcoidosis. The non-infectious aetiology of GLILD is underlined; in addition, this form of ILD often coexists with autoimmune manifestations [18]. Granulomatous-lymphocytic interstitial lung disease is mainly diagnosed in adult patients; however, there are reports of the diagnosis in children and even infants $[19,20]$, as well as its occurrence in other PIDs, e.g. DGS [21]. The incidence of bronchiectasis in XLA patients is almost $32 \%$ and accounts for up to $3 \%$ of cases of childhood bronchiectasis [22]. It is worth highlighting that we diagnosed ILD in 1 patient with XLA, although according to the literature, ILD also appears to be very rare or absent in this condition [4]. Interestingly, the incidence of pulmonary complications appears to be higher in CVID than $\mathrm{XLA}$, and it is probably related to factors other than antibody deficiency $[15,23]$.

Recurrent infections are a major factor inducing serious respiratory complications. Viral-especially caused by Herpes and fungal lung infections are indicated in the literature as possible risk factors for ILD [4, 5, 24, 25]. However, immune dysregulation has recently been mentioned as the leading cause of ILD. Autoimmunity in PIDs may be triggered by different mechanisms, including defects of tolerance to self-antigens or persistent stimulation resulting from the inability to eradicate antigens $[18,26,27]$. Thus far, several studies have revealed a correlation between autoimmunity and the development of ILD [26, $28,29]$. This differs from the findings presented here, in which autoimmune diseases occurred with similar frequency in all groups. Nevertheless, the spectrum of reported autoimmune diseases is comparable [26, 27]. Conversely, numerous reports point out recurrent pneumonia as the main cause of bronchiectasis. The association with persistent inflammation in the airways also suggests older age of patients $[17,29]$.

In accordance with the present results, previous studies have demonstrated that splenomegaly and lymphadenopathy are often present at the diagnosis of ILD, which is associated with the autoimmune dysregulation mentioned above. This study's results show that sinusitis is the most common accompanying disease, which is consistent with the literature [1-3]. Chronic inflammation and bacterial colonization of the paranasal sinuses may be a starting point for diseases involving the lower respiratory tract; hence, optimal pulmonary treatment should always encompass the upper airways [30]. Over a fifth of the children enrolled in this study were diagnosed with asthma, and interestingly all these children presented radiological features of ILD. It should be emphasized that symptoms characteristic of asthma, such as a cough and exercise intolerance, may also be the first signs of chronic 
lung disease. Therefore, in any case of suspected asthma in a child with PID, a detailed differential diagnosis should be carried out [31].

Despite the suggested distinct aetiology of ILD and bronchiectasis, the coexistence of both diseases is reported in a large group of patients. Maglione et al. [28] found that in adults with CVID, bronchiectasis may coexist in as much as $67 \%$ of ILD cases. Furthermore, the analysis of CVID patients by Weinberger et al. [23] showed the relationship between ILD and the occurrence of pneumonia and bronchiectasis. To date, there has been no detailed investigation of complex lung disease in immunodeficient children. It should be mentioned that radiological imaging, especially HRCT, is not routinely obtained in children with predominant antibody deficiencies, despite the recommendations [5, 32]. Moreover, increased radiosensitivity occurring in some PIDs (e.g. AT, NBS, CVID, LRBA deficiency, and hiper-IgM syndrome) is a contraindication to X-ray imaging. Nevertheless, the recommendations for the diagnosis and management of chronic pulmonary disease and AT indicate the advantages of performing low-dose CT over delaying the diagnosis of chronic pulmonary disease [11].

Our observations may support the hypothesis that, despite IgRT, some patients develop chronic lung disease, which matches earlier reports [15]. Moreover, serious pulmonary complications may progress despite adequate serum IgG concentration. Therefore, active and precise screening is mandatory even in "well-controlled" patients [30]. In our study a quarter of the patients were not treated pharmacologically before the diagnosis, which may indicate the insidious and asymptomatic development of chronic lung disease, also highlighted in recent reports [33]. These outcomes confirm the fact that appropriate management of PIDs remains an unresolved issue.

The study's major limitation is the relatively small group of patients, resulting from a low prevalence of PIDs. Secondly, the different imaging techniques used in assessing lung complications make these findings less generalizable. In addition, due to the retrospective nature of the study, further prospective follow-up studies are required.

\section{CONCLUSIONS}

The study has shown that serious pulmonary complications occur in immunodeficient children at all ages and are connected with different PIDs. Although the aetiologies of ILD and bronchiectasis differ, a significant number of the patients present features of both diseases. Regular clinical evaluation, supported by the results of additional tests, including radiological findings, is crucial for the early diagnosis of chronic lung disease. The present study lays the groundwork for future research into optimal management in the prophylaxis of serious pulmonary complications in children with PIDs.

\section{DISCLOSURE}

The authors declare no conflict of interest.

\section{REFERENCES}

1. Buckley RH. Pulmonary complications of primary immunodeficiencies. Paediatr Respir Rev 2004; 5: S225-S233.

2. Jesenak M, Banovcin P, Jesenakova B, et al. Pulmonary manifestations of primary immunodeficiency disorders in children. Front Pediatr 2014; 2: 77.

3. Yazdani R, Abolhassani H, Asgardoon M, et al. Infectious and Noninfectious Pulmonary Complications in Patients With Primary Immunodeficiency Disorders. J Investig Allergol Clin Immunol 2017; 27: 213-224.

4. Schussler E, Beasley MB, Maglione PJ. Lung Disease in Primary Antibody Deficiencies. J Allergy Clin Immunol Pract 2016; 4: 10391052.

5. Tarzi MD, Grigoriadou S, Carr SB, et al. Clinical Immunology Review Series: An approach to the management of pulmonary disease in primary antibody deficiency. Clin Exp Immunol 2009; 155: 147-155.

6. Gallucci M, di Palmo E, Bertelli L, et al. A pediatric disease to keep in mind: diagnostic tools and management of bronchiectasis in pediatric age. Ital J Pediatr 2017; 43: 117.

7. Haidopoulou K, Calder A, Jones A, et al. Bronchiectasis secondary to primary immunodeficiency in children: longitudinal changes in structure and function. Pediatr Pulmonol 2009; 44: 669-675.

8. ESID - European Society for Immunodeficiencies, https://esid.org/ Working-Parties/Registry-Working-Party/Diagnosis-criteria (accessed 18 December 2020).

9. Tangye SG, Al-Herz W, Bousfiha A, et al. Human Inborn Errors of Immunity: 2019 Update on the Classification from the International Union of Immunological Societies Expert Committee. J Clin Immunol 2020; 40: 24-64.

10. van der Burg M, Gennery AR. Educational paper. The expanding clinical and immunological spectrum of severe combined immunodeficiency. Eur J Pediatr 2011; 170: 561-571.

11. McGrath-Morrow SA, Gower WA, Rothblum-Oviatt C, et al. Evaluation and management of pulmonary disease in ataxia-telangiectasia. Pediatr Pulmonol 2010; 45: 847-859.

12. Schroeder SA, Swift M, Sandoval C, et al. Interstitial lung disease in patients with ataxia-telangiectasia. Pediatr Pulmonol 2005; 39: 537-543.

13. Deerojanawong J, Chang AB, Eng PA, et al. Pulmonary diseases in children with severe combined immune deficiency and DiGeorge syndrome. Pediatr Pulmonol 1997; 24: 324-330.

14. Freeman AF, Olivier KN. Hyper-IgE Syndromes and the Lung. Clin Chest Med 2016; 37: 557-567.

15. Patrawala M, Cui Y, Peng L, et al. Pulmonary disease burden in primary immune deficiency disorders: Data from USIDNET Registry. J Clin Immunol 2020; 40: 340-349.

16. Ramzi N, Jamee M, Bakhtiyari M, et al. Bronchiectasis in common variable immunodeficiency: A systematic review and meta-analysis. Pediatr Pulmonol 2020; 55: 292-299.

17. van de Ven AJM, de Jong PA, Hoytema van Konijnenburg DP, et al. Airway and interstitial lung disease are distinct entities in paediatric common variable immunodeficiency. Clin Exp Immunol 2011; 165: 235-242.

18. Uzunhan Y, Jeny F, Kambouchner M, et al. The lung in dysregulated states of humoral immunity. Respir Int Rev Thorac Dis 2017; 94 : 389-404. 
19. Nademi Z, Davies G, Devlin L, et al. Granulomatous lymphocytic interstitial lung disease (GLILD) in children. Arch Dis Child 2018; 103: A58.

20. Adeleye A, Kelly M, Wright NA, et al. Granulomatous lymphocytic interstitial lung disease in infancy. Can Respir J 2014; 21: 20-22.

21. Sood AK, Funkhouser W, Handly B, et al. Granulomatous-lymphocytic interstitial lung disease in 22q11.2 deletion syndrome: a case report and literature review. Curr Allergy Asthma Rep 2018; 18: 14.

22. Li AM, Sonnappa S, Lex C, et al. Non-CF bronchiectasis: does knowing the aetiology lead to changes in management? Eur Respir J 2005; 26: 8-14.

23. Weinberger T, Fuleihan R, Cunningham-Rundles C, et al. Factors Beyond Lack of Antibody Govern Pulmonary Complications in Primary Antibody Deficiency. J Clin Immunol 2019; 39: 440-447.

24. Kellner ES, Fuleihan R, Cunningham-Rundles C, et al. Cellular Defects in CVID Patients with Chronic Lung Disease in the USIDNET Registry. J Clin Immunol 2019; 39: 569-576.

25. Vidal S, de la Horra C, Martín J, et al. Pneumocystis jirovecii colonisation in patients with interstitial lung disease. Clin Microbiol Infect Off Publ Eur Soc Clin Microbiol Infect Dis 2006; 12: 231-235.

26. Allenspach E, Torgerson TR. Autoimmunity and primary immunodeficiency disorders. J Clin Immunol 2016; 36 Suppl 1: 57-67.

27. Giardino G, Gallo V, Prencipe R, et al. Unbalanced immune system: immunodeficiencies and autoimmunity. Front Pediatr 2016; 4: 107.

28. Maglione PJ, Overbey JR, Cunningham-Rundles C. Progression of common variable immunodeficiency interstitial lung disease accompanies distinct pulmonary and laboratory findings. J Allergy Clin Immunol Pract 2015; 3: 941-950.

29. Maglione PJ, Overbey JR, Radigan L, et al. Pulmonary radiologic findings in common variable immunodeficiency: clinical and immunological correlations. Ann Allergy Asthma Immunol Off Publ Am Coll Allergy Asthma Immunol 2014; 113: 452-459.

30. Baumann U, Routes JM, Soler-Palacín P, et al. The Lung in Primary Immunodeficiencies: New Concepts in Infection and Inflammation. Front Immunol 2018; 9: 1837.

31. Truong T. The overlap of bronchiectasis and immunodeficiency with asthma. Immunol Allergy Clin North Am 2013; 33: 61-78.

32. Touw CML, van de Ven AA, de Jong PA, et al. Detection of pulmonary complications in common variable immunodeficiency. Pediatr Allergy Immunol Off Publ Eur Soc Pediatr Allergy Immunol 2010; 21: 793-805.

33. Pac M, Bielecka T, Grzela K, et al. Interstitial lung disease in children with selected primary immunodeficiency disorders - a multicenter observational study. Front Immunol 2020; 11: 1950. 